\title{
MICROSOFT PIXELSENSE - INTERACTIVE SURFACE COMPUTING PLATFORM FOR SAMSUNG SUR40
}

\author{
Madhura Mhatre $^{1}$, Siddhi Choudhari ${ }^{2}$, Nishtha Chourasia ${ }^{3}$ \\ ${ }^{1,2,3}$ L.T.C.O.E, Navi Mumbai \\ ${ }^{1}$ madhuramhatre95@gmail.com, ${ }^{2}$ siddhichoudhari95@gmail.com
}

\begin{abstract}
:
Microsoft PixelSense is an interactive surface computing platform that allows a richer visual experience and provides a radical new way to manipulate data by introducing multi-touch gestures. It allows users to interact with GUI without the use of mouse and a keyboard, providing a natural user interface. It contains the features of both PC and a tablet. It recognizes touch and nondigital objects on the screen's surface and is designed to sync instantly with any device that touches its from toothbrush to cell phones, thus merging the real and the virtual world. It is built with wireless transceivers, Bluetooth and Wi-Fi. It utilizes raw vision data and has the ability to recognize fingers, tags and blobs. It provides a new area of business opportunities which can be used in grand hotels, airports, gaming zones, bing maps, real estate, colossal casino's and in restaurants. It is a creative way to break down the old traditional barriers to technology
\end{abstract}

Keywords: Blobs-Binary large object, colossal-large,Bing maps-interactive map.

\section{INTRODUCTION}

Nowadays the rapid development and research of information technology is playing an increasingly important role in personal lives of people Computers, digital information, software's, mobiles, tablets are the constituents of the information age which can be found every where therefore it is difficult to survive in the modern world without any of these gadgets. Every piece of technology that is made requires little or no skill at all. Instead of operating all the devices separately one can perform all the functions on the same platform simultaneously therefore saving time and energy.

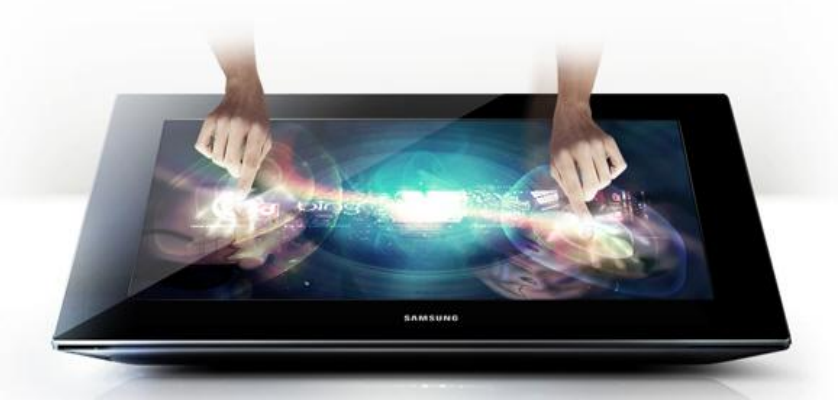

Microsoft PixelSense Is a latest technology put forward by Microsoft which any computer literate can use without being a power user. It was initially released on April $17^{\text {th }}$ 2008 by Microsoft. The latest version of Microsoft Surface was renamed as Microsoft PixelSense on June $18^{\text {th }} 2012$, and is currently being used in Samsung SUR40.

This remarkable technology enables Microsoft and Samsung to reduce the device size from 22 inch $(56 \mathrm{~cm})$ to 4 inch $(10 \mathrm{~cm})$ in thickness. Which allows the device to be placed horizontally or mounted vertically. It makes the experiences that change the way people collaborate and connect with a stunning 360-degree interface. PixelSense is flamboyant and profoundly smart technology which can react to more than 50 points of contact simultaneously, thus several users can use the same device for heightened collaboration and connection. PixelSense was always the name of Microsoft's optical sensor for the SUR40. The multi-user featured input is powered by an Infrared Sensor System.

Applications in Microsoft PixelSense are built using the Microsoft Surface 2.0 SDK, and are ideal for any scenario in which users need to interact together on a single device. The focus of PixelSense is on creating real connectionswhether it's connecting customers with information and each other, or connecting the device to other devices.

This technology promotes easy digitization and works on an efficient collaboration of hardware and software.

\section{MAIN}

Samsung SUR40 with Microsoft PixelSense is a thin LCD of display size 40 inch $(101.6 \mathrm{~cm})$ diagonally and a display resolution of $1920 \times 1080-16: 9$ aspect ratio. PixelSense recognizes and response to touch with the help of five nearinfrared (IR) cameras that can see fingers and objects placed on the display. The physical dimensions of the device are $(\mathrm{L} \times \mathrm{W} \times \mathrm{H}): 42.7 \times 27.5 \times 4$ inch $(108.5 \times 69.9 \times 10.2 \mathrm{~cm})$ and weighs $80 \mathrm{lbs}(36 \mathrm{~kg})$. The screen consist of a protective LCD glass panel with many integrated sensors and optical sheets consisting of PixelSense processing units along with backlight that has visible and infrared LED's. The sleek components of device rest on embedded pc housing for better longevity. It is enhanced with $\mathrm{AMD}$ (advanced micro devices) Athlon II X2 $2.9 \mathrm{GHz}$ dual-core processor as the central processing unit and, AMD Radeon HD 6570M - 1 
GB GDDR5 as the Graphics processing unit. Networking plays a vital role in development therefore for better connectivity the devices is powered with Wi-Fi 802.11n, Bluetooth, and Ethernet 10/100/1000. For better utility it is provided with extensions ports such as HDMI input \& output, S/PDIF 5.1 digital audio surround sound out, RCA analog component audio out, $3.5 \mathrm{~mm}$ TRS (stereo minijack) audio out, 4 USB ports. The operating system used is Windows 7 professional for embedded systems (64-bit). The storage capacity provided is 320 GB HDD (Hard disk drive) storage capacity and has a memory space of 4GB DDR3.The cost ranges from $\$ 8,400$ USD.

The PixelSense platform is basically the combination of vision input technology and creative display. The software development kit used is Microsoft Surface 2.0. The technology allows the non digital objects to be used as input devices .Object recognisation corresponds to the device's, ability to recognize the presence and orientation of tagged objects placed on the screen .

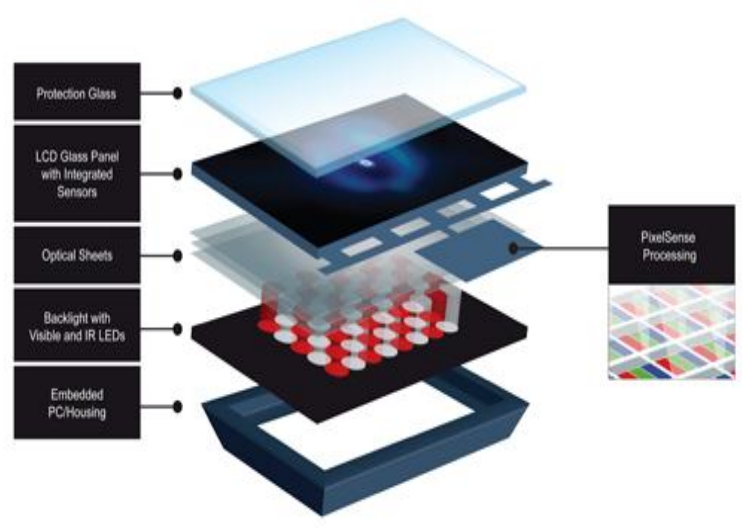

As soon as anything comes in contact with the screen the infrared back light unit flashes light at the place of contact. The light was reflected to multiple infrared cameras allowing it to sense the object's coordinates. The sensors covert the light signal into an electrical value. All the values collected are then processed to re-create a picture of what actually is placed on the table top. And then using image processing techniques the picture is analyzed, and the output is sent to the PC. And interestingly all this process in done within fraction of seconds.

\begin{tabular}{|c|c|c|}
\hline FUNCTIONALITY & MICROSOFT SURFACE 1.0 & $\begin{array}{l}\text { SAMSUNG SUR40 FOR } \\
\text { MICROSOFT SURFACE }\end{array}$ \\
\hline Form factor usage & Tables and counters & Tables, counters, kiosks, and walls \\
\hline Display+vision input technology & Rear projection DLP w/cameras & Thin LCD w/pixelsense" technology \\
\hline Price & S12,500 USD commercial & MSRP starting at 57,600 USD \\
\hline Weight & $198 \mathrm{lbs}$. & $87 \mathrm{lbs}$. \\
\hline Physical dimensions $(\mathrm{a} \times \mathrm{W} \times \mathrm{H})$ & $42.5 \times 27 \times 21 \mathrm{in}$. & $42.7 \times 27.5 \times 4 \mathrm{in}$ \\
\hline CPU & $\begin{array}{l}\text { Intel' Core" } 2 \text { Duo } \\
2.13 \text { GHz processor }\end{array}$ & $\begin{array}{l}\text { AMD Athlon" } 11 \text { X2 } \\
\text { 2.9 } \mathrm{GHz} \text { Dual-Core processor }\end{array}$ \\
\hline Graphics (GPU) & ATI Radeon" X1650 - 256 MB & $\begin{array}{l}\text { AMD Radeon" HD } 6570 \mathrm{M}-1 \text { GB } \\
\text { GDDR5 }\end{array}$ \\
\hline Memory & 2 GB DDR2 & $4 G B-8 G B$ DDR3 \\
\hline Storage (hard drive) & 160 GB HDD - 5400 RPM & 320 GB HDD - 7200 RPM \\
\hline Display size & $30 \mathrm{in.}$ & $40 \mathrm{in.}$ \\
\hline Display resolution & $1024 \times 768-4: 3$ aspect ratio & $\begin{array}{l}1920 \times 1080 \text { - } 1080 \text { p HD } 16: 9 \text { aspect } \\
\text { ratio }\end{array}$ \\
\hline Extensions (ports) & $\begin{array}{l}\text { XGA (DE-15) video out } \\
\text { RGB analog component video out } \\
\text { RCA analog component audio out } \\
4 \text { USB ports }\end{array}$ & $\begin{array}{l}\text { HDMl input } \& \text { output } \\
\text { SPDIF } 5.1 \text { digital surround sound out } \\
\text { RCA analog component audio out } \\
3.5 \mathrm{~mm} \text { TRS (stereo mini-jack) audio out } \\
4 \text { USB Ports }\end{array}$ \\
\hline Networking & Wi-Fi 802.11g, Bluetooth, and Ethernet & Wi-Fi 802.11n, Bluetooth, and Ethernet ${ }^{16}$ \\
\hline
\end{tabular}

\section{ADVANTAGES}

\section{Following are the various advantages:-}

[1]. Multi-touch contact refers to the ability to have multiple contact points with an interface, unlike with a mouse, where there is only one cursor.

[2]. Object recognition refers to the device's ability to recognize the presence and orientation of tagged objects placed on top of it.

[3]. Multi-user experience is a benefit of multi-touch: several people can orient themselves on different sides of the surface to interact with an application simultaneously.

[4]. It requires very less skill or no skill for its operation.

[5]. It is multi-purpose and easy to clean and maintain.

[6]. Direct interaction refers to the user's ability to simply reach out and touch the interface of an application in order to interact with it.

\section{APPLICATIONS}

[1]. In Financial services Industry to increase sales by illustrating and comparing different financial products and plns.

[2]. In Retail outlets to deliver social shopping experiences to customers.

[3]. In hospitality to increase guest satisfaction and operational efficiencies.

[4]. In Educational sector for delivering unique learning experience that utilizes more of the senses.

\section{Creating Applications for Microsoft PixelSense}

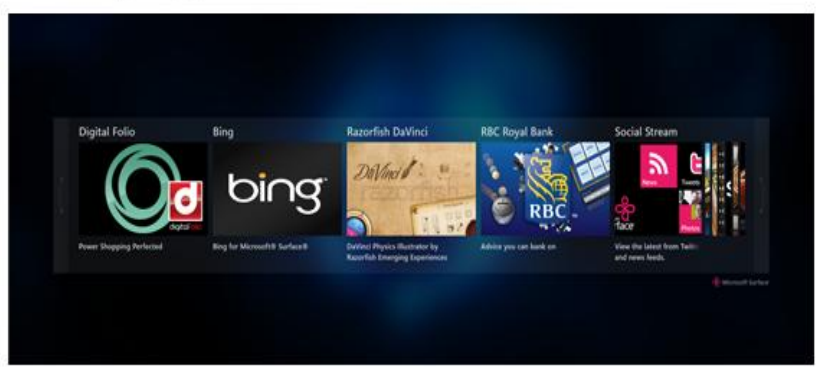

\section{USEFUL TOOLS PRESENT:}

[1]. Infusion : Royal Bank of Canada Application.

[2]. Vectorform LLC : Galatic Allaince

[3]. T-Systems Multimedia Solutions : RedBull Interactive

[4]. Sevensteps : The Real Estate Agent

[5]. Touchtech AB : Tourist Guide

[6]. Razorfish : DaVinci

[7]. Nsquared : seven new surface application

[8]. IntuiLab : IntuiFace Presentation

[9]. LieberLieber Software : GmbH POI Explorer

[10]. Customer Solutions Group LLC : Digital Folio

[11]. Ergonomidesign and Connecta : Sonicspree

[12]. Aspect Software Inc. : The Voyages of Columbus

[13]. Brown University: Garibaldi

[14]. AFTER-MOUSE: Photos

[15]. AFTER-MOUSE: Paint'Touch 


\section{FUTURE SCOPE}

The PixelSense table is a powerful tool within intimate point of sale. The product features easy, effective and interactive way to acess information. In this way you can give an opportunity to the user to engage with product rather than sales people and catalogues. Apart from sales, it has a wide scope in Gaming World. Games can be played in a new fashion altogether with multi-touch and multi-user feature.

Machine, Automobile and Fashion designing can be done more creatively using the gesture and 360 degree interface feature. In Education sector for better understanding and visualization of topics such as Earth Terrain, Science, Information Technology etc.

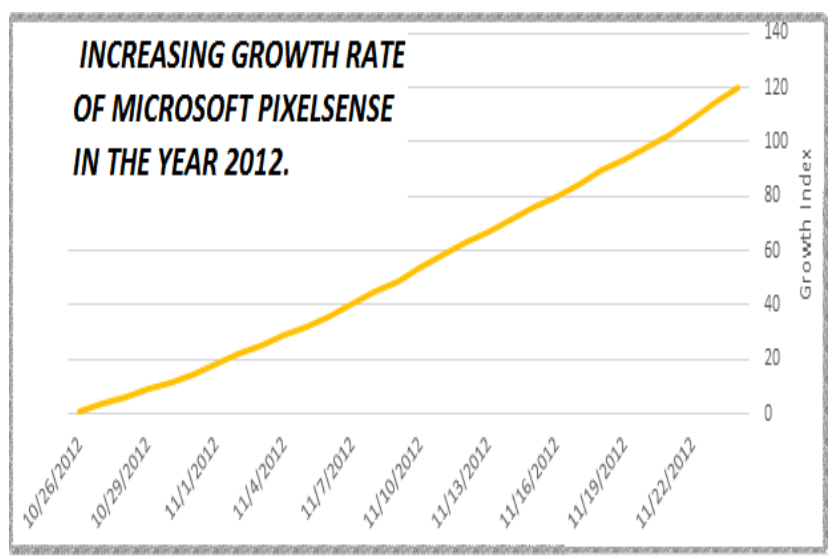

\section{CONCLUSION}

Hence conclude that MICROSOFT PIXELSENSE is:

A perfect platform for creative and innovative minds to transform their ideas into reality. It is a convenient technology which has the potential to replace various other devices. It is a great tool in hand for better accessing and exploring information. It amazingly merges the real and the virtual world. Its efficiency and the interaction level makes it quick, easy and user-friendly to work with.

\section{REFERENCES:}

[1]. https://www.google.co.in/search?q=pixelsense+logo\& biw $=1280 \&$ bih $=663 \&$ source $=1$ mms\&sa $=X \&$ ei $=B K 7 R$ VIOmKon58QXBq4CgCA\&v

[2]. http://en.wikipedia.org/wiki/Microsoft_PixelSense

[3]. https://www.youtube.com/user/MSPixelSense

[4]. http://www.microsoft.com/enus/pixelsense/default.aspx

[5]. http://www.microsoft.com/enus/pixelsense/whatsnew.aspx

[6]. http://www.samsung.com/au/business/businessproducts/smart-signage/specialisedsolutions/LH40SFWTGC/XY 